\title{
Factores Favorecedores y Obstaculizadores de la Transferencia de la Formación del Profesorado en Educación Superior
}

\author{
Favouring and Hindering Factors in the Transfer of Teachers \\ Training Process in Higher Education
}

Elena Cano *

Universitat de Barcelona

\begin{abstract}
La formación del profesorado constituye un recurso para la mejora de la calidad educativa. Por ello es necesario estudiar si se transfiere a las aulas e impacta en las instituciones y en el conjunto del sistema. Este artículo tiene por objetivo analizar los factores que favorecen y dificultan dicho proceso a partir de realizar un sistemático análisis documental de las investigaciones sobre transferencia de la formación realizadas en los últimos años. El diseño de la evaluación junto a la planificación de la acción formativa o el compromiso institucional, junto con la autoeficacia o la percepción de la capacidad de transferir, son los principales elementos que favorecen el traspaso de la formación al trabajo diario de los docentes. Por el contrario, la falta de apoyo de superiores e iguales o la falta de conexión entre la formación y los planes de centro son los factores que actúan como mayores obstáculos para la transferencia de la formación. El estudio determina que evaluar la transferencia es un proceso complejo y costoso por la necesidad de realizarse de forma diferida en el tiempo, de implicar a diversos agentes y de recoger datos que puedan evidenciar las mejoras generadas por la formación.
\end{abstract}

Descriptores: Transferencia de la formación, Formación continua, Finalidades de la formación, Evaluación de programas, Profesorado.

Teacher training is a resource for improving the quality of education To consider if training is transferred to the classroom and if it is impacting in institutions and in the whole system is a must. This article analyses the factors that foster and hinder this process from a systematic documentary analysis of last years' developed research on transfer of training. The evaluation design, the training planning, the institutional commitment, together with the self-efficacy or ability to transfer selfperception, are the main elements that favor the transfer of training to the daily work of teachers. On the contrary, the absence of support from heads and peers and the lack between training and institutional plans are the major obstacles to the transfer of training. The study determines that transfer assessment is a complex and expensive process because it has to be done delayed in time, it has to involve different stakeholders and it has to collect data for proving the benefits that it has got.

Keywords: Transfer of training, Continuous training, Training objectives, Program evaluation, Teachers.

*Contacto: ecano@ub.edu

ISSN: $1696-4713$

www.rinace.net/reice/
Recibido: $\quad 16$ de junio 2015

$1^{\text {a }}$ Evaluación: 21 de agosto de 2015

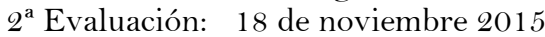

Aceptado: $\quad 15$ de enero 2016 


\section{Introducción}

En tiempos de crisis parece, aunque no se dispone de datos claros, que uno de los recortes más severos han sido las actividades de formación. Así lo han denunciado tanto

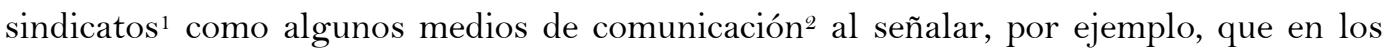
presupuestos de 2012 la partida que más cayó fue la dedicada a la formación permanente del profesorado (cursos de actualización), que pasó de 52 millones a 4 (-91\%).Si la formación se considera como algo accesorio o complementario a la tarea diaria de los docentes, podría estimarse como algo prescindible. Ahora bien, si la formación se entiende como un recurso necesario para mantenerse actualizado tanto en los saberes de cada disciplina como en las didácticas de cada una de ellas, y se concibe más como inversión que como gasto, recortar en formación supone, a todas luces, una política errónea, que puede tener exiguos ahorros a corto plazo y enormes déficits a medio y largo plazo. Bonal (2015) recuerda los efectos devastadores que los recortes de los derivados de los programas de ajuste estructural tuvieron en los 80's sobre la calidad educativa. Por su parte, Melgarejo (2013) muestra cómo Finlandia, país con altos resultados en pruebas PISA y con un modelo educativo de referencia, en lo que invierte más es en la formación inicial y continua del profesorado.

Hoy más que nunca los retos que tiene que enfrentar el sistema educativo sólo pueden lograrse con unos profesionales competentes, actualizados, innovadores y capaces de trabajar en equipo con otros agentes educativos y sociales. Sin embargo, también es cierto que las políticas de formación deben de basarse en macro análisis de sistema y meso análisis de centro que identifiquen las necesidades de forma rigurosa, de modo que se emprendan acciones que resulten estratégicas para lograr los objetivos del sistema educativo y que contribuyan al desarrollo de planes estratégicos y de mejora de los centros. Con ello quizás se evite la impresión que tiene cierta parte de la población de que la formación obedece más a modas o corrientes (diversas e incluso, en ocasiones, opuestas) acerca de lo que es necesario (Sanfabián, Belver y Álvarez, 2014). Por otra parte, la inversión en formación del profesorado debiera también financiar aquellas modalidades formativas que se han revelado como más eficientes. En este sentido, el movimiento de mejora de la escuela (Murillo, 2003) ha mostrado los beneficios que para lograr el cambio educativo posee la formación en centros. También la OCDE (2005) en el informe en el que defendía que "la docencia importa" alertaba de la necesidad de articular de forma más ajustada la formación del profesorado, su perfeccionamiento profesional y las necesidades de los centros educativos, sugiriendo una mayor responsabilidad de los centros en la formación y la gestión personal del profesorado para alinear mejor formación y planes de centro.

\section{Revisión de la Literatura}

Si bien se dispone de prolija literatura sobre modelos y modalidades formativas y sobre la importancia de la formación del profesorado, inicial y permanente, para el desarrollo profesional, la evaluación de la formación ha sido un tema menos abordado y su tratamiento se ha realizado más desde un plano teórico que desde con estudios

\footnotetext{
${ }^{1}$ http://www.feteugt.es/data/Upload/GABrecortes_partidas_presupuestarias_educacion.pdf

${ }^{2}$ http://www.elmundo.es/elmundo/2012/04/03/espana/1333453157.html
} 
empíricos, por la dificultad que entrañan. En caso de realizarse, éstos han abordado los efectos o resultados de la formación en términos de percepciones de los usuarios, con lo que la evaluación de la formación en su globalidad parece ser un tema pendiente.

\subsection{La evaluación de la formación, un tema pendiente}

En la mayoría de países de la UE, administrativamente se articulan procesos para rendir cuentas de la formación realizada (Eurydice, 2006) en tanto se informa del grado de ejecución de los planes de formación previstos. Sin embargo, ¿existen estudios sobre los efectos que las diversas modalidades formativas tienen? Es decir, ¿se evalúa el resultado de las diferentes acciones con el fin de tomar decisiones fundamentadas en datos? En términos generales, las diversas administraciones y unidades encargadas de formación de profesorado evalúan un único tipo de resultados: la satisfacción de los participantes con la actividad desarrollada y, en algunos casos, la percepción que el propio formador/a tiene de cómo ha funcionado la actividad formativas. También es cierto que existen algunas experiencias que tratan de averiguar los efectos de modalidades diversas (Pineda, 2012) o que tratan de incorporar otros niveles de resultados (Quesada, Espona, Ciraso y Pineda, 2015) pero su coste, la dimensión temporal extensa y la necesaria implicación de diversos agentes, las hacen poco viables y en ocasiones se asocian con investigaciones llevadas a cabo desde la universidad o con estudios piloto asociados a proyectos de innovación o de investigación que, una vez acabado el proyecto, dejan de ponerse en práctica.

En nuestro entorno los análisis más relevantes al respecto son los realizados por Pineda (Pineda 2000, 2002, 2011; Pineda y Sarramona, 2006), que ha desarrollado tanto estudios teóricos sobre modelos de evaluación como estudios empíricos de evaluación (Pineda, Moreno, Úcar y Belvis, 2010; Pineda, Belvis, Moreno, Durán y Úcar 2011). Algunos otros análisis recientes sobre la evaluación de la transferencia de la formación son los de Gairín (2010); Tejada y Ferrández (2007) o Tejada, Ferrández, Jurado, Mas, Navío y Ruiz (2008). En el ámbito universitario, destacan los trabajos de Feixas (2013) y Feixas, Fernández, Lagos, Quesada y Sabaté (2013), así como la aportación de Fernández, Guisasola, Garmendia, Alkorta y Madinabeitia (2013). Mención especial merece el reciente monográfico coordinado por Feixas (2015) acerca del tema. Casi todos los estudios hacen referencia al modelo más popular respecto a la evaluación de los diversos niveles de resultados de la formación, que es el formulado por Kirkpatrick y Kirkpatrick (2000) aunque también al de Holton (1996, 2005).

La propuesta de Kirkpatrick y Kirkpatrick (2000) para evaluar los resultados de un programa formativo establece cuatro niveles: (1) satisfacción o reacción; (2) aprendizaje (o desarrollo de competencias); (3) transferencia y (4) impacto. La satisfacción con la actividad formativa parece ser ampliamente recogida en casi todos los programas formativos . Puede centrarse en la opinión de los participantes y/o contrastarla con la del formador/a. Puede realizarse en papel o, cada vez más, en línea, aunque ello ocasiones dificultades en la recogida de datos. Puede realizarse con instrumentos cerrados (escalas, check-list e incluso rúbricas) o de forma más cualitativa (que es menos usual puesto que normalmente no se centra en la comprensión sino en la recogida de evidencias objetivas para el rendimiento de cuentas y requiere de indicadores numéricos). Pero, en cualquier caso, este primer nivel suele estar resuelto. El aprendizaje derivado de los procesos formativos ha sido poco considerado en la formación permanente del profesorado, quizás por resistencias culturales o por la propia 
organización y logística de la formación permanente. Pueden recogerse evidencias de aprendizaje individuales o colectivas elaboradas a lo largo de la formación pero resulta poco usual que se solicite acreditar el aprendizaje obtenido por otras vías. Por otra parte, si se trata de formaciones colectivas y ligadas a los proyectos de centro, puede no ser necesario evaluar este nivel si se evalúa el nivel siguiente (transferencia) puesto que transferir, si no se hace de forma mecánica e irreflexiva, indica que ha habido aprendizaje. Sin embargo, la transferencia o la medida en que los aprendizajes adquiridos durante la formación se aplican en la práctica aún ha sido, si cabe, menos contemplada y, finalmente, el impacto también ha sido menos considerado en la evaluación de programas. La transferencia, como recogen Feixas, Lagos, Fernández y Sabaté (2015) es el grado en que los participantes aplican eficazmente el conocimiento, las habilidades y las actitudes requeridas en un contexto de trabajo o el conjunto de evidencias que muestran que lo que se ha aprendido realmente está siendo utilizado en el trabajo para el cual fue pensado, mientras que el impacto es la repercusión del plan de formación a nivel individual, colectivo u organizativo. Es decir, se trata de evaluar "ex-post" los cambios observables que una acción formativa tiene, sean éstos cuantitativos, traducibles en valores monetarios, o, en nuestro caso, cualitativos, no traducibles en términos económicos sino en mejoras de diferente tipo en las actitudes, el conocimiento y las habilidades de los docentes; en el comportamiento y en las prácticas docentes; en la experiencia de aprendizaje del estudiante, etc. (Parsons, Hill, Holland y Willis, 2012).

Como indican los propios autores Kirkpatrick y Kirkpatrick (2010), las organizaciones dedican más del $72 \%$ de la evaluación a los niveles 1 y 2 y se trabaja muy poco en los niveles 3 y 4. De modo análogo, Pineda (2000) halla en su estudio que el 100\% de las organizaciones que analiza evalúa la satisfacción del participante con la formación, evaluación que se aplica a casi la totalidad de las acciones formativas $(90 \%)$, y para la que se utiliza fundamentalmente el cuestionario (95\%), junto con los contactos informales $(50 \%)$ y las entrevistas (23\%). La transferencia de la formación al puesto de trabajo, en cambio, se evalúa solo en el $62 \%$ de las empresas y además sobre menos del $40 \%$ de las acciones formativas. Los instrumentos que se utilizan con más frecuencia son las entrevistas a los supervisores (70\%), seguidas de la observación (60\%) y de la evaluación del desempeño (40\%), y los agentes que realizan esta evaluación suelen ser el departamento de formación y el supervisor del participante. Los motivos que las organizaciones alegan para no realizar este tipo de evaluación son la dificultad para medir determinados factores y la falta de recursos y conocimientos sobre el tema. Por lo tanto, parece que el actual reto reside en evaluar la transferencia de la formación.

\section{2. ¿Qué es transferencia de la formación?}

Si se realiza una búsqueda sobre estudios de transferencia, aparecen más documentos acerca de transferencia del aprendizaje que acerca de transferencia de la formación propiamente. Quizás por eso, en primer lugar, hay voces que identifican la transferencia de la formación con la transferencia del aprendizaje (Transfer of learning), aspecto largamente estudiado en su día por Thorndike y que se entiende como el traspaso de habilidades y conocimientos adquiridos en una situación a otra situación, el modo en que los aprendizajes anteriores influyen o se aplican en aprendizajes posteriores o el uso de aprendizajes en general. El libro de Haskell (2001) y más recientemente la investigación de Haverila Myllylä y Torp (2009) constituyen buenos ejemplos de este tipo de estudios Sin embargo, si nos circunscribimos a la transferencia de la formación, la aportación más conocida es la de Baldwin y Ford, quienes la identifican con el grado en el que los 
alumnos (o participantes en una actividad formativa) aplican efectivamente los conocimientos, habilidades y actitudes obtenidas en el contexto formativo (o de entrenamiento) al puesto de trabajo (Baldwin y Ford, 1988, p. 63). En el campo de la formación del profesorado destacan los trabajos de Evans, Hodkinson, y Unwin (2002) y de Evans, Hodkinson, Rainbird y Unwin (2006).

Según los propios Baldwin y Ford para que se dé transferencia ha de producirse una aplicación generalizada en el contexto de trabajo (Generalización) y una persistencia en el tiempo (Mantenimiento). La generalización significa, como ya indicaron Tejada et al. (2008) que los aprendizajes se utilizan en contextos, personas o condiciones diferentes a aquellos en los que fueron adquiridos, es decir, implica trasladarlo a escenarios diferentes al contexto formativo. Por otra parte, el mantenimiento, como señalan Blume Ford, Baldwin y Huang (2009) y De Rijdt, Stes, van der Vleuten y Dochy (2013) implica la sostenibilidad en el tiempo, la aplicación continuada.

A estos dos aspectos Ford y Weissbein (1997), citados por Cordero y Ornelas (2014), añaden un tercer requisito: adaptabilidad, que supone ajustar el conocimiento a nuevas situaciones, lo cual implica la aplicación no mecánica sino contextualizada. La adaptabilidad se refiere al desarrollo de un comportamiento estratégico donde los conocimientos adquiridos actúan únicamente como principios orientadores para ser ajustados a nuevos contextos. Esta noción de adaptabilidad encaja con las propuestas de los diseños basados en competencias que se realizan actualmente en tanto que requiere analizar y problematizar una situación y evocar, movilizar y combinar los saberes que se disponen para aplicarlos en contextos diversos y lograr resolver eficientemente diferentes situaciones, superando aplicaciones mecánicas o estereotipadas.

Por ello, hay algunos aspectos que parecen claros si se desea evaluar la transferencia de la formación: que debe de realizarse de forma diferida en el tiempo y que debe de contemplar la aplicación de forma no mecánica, sino ajustada a los diversos contextos de intervención. Por otra parte, para evaluar dicha transferencia, se pueden emplear diversas fuentes, en función de los recursos disponibles para evaluar el programa formativo e incluso de la madurez del colectivo implicado: se puede recoger la percepción de los participantes de si han transferido (lo cual sería una medida indirecta); se puede recoger la opinión de iguales y superiores respecto a los cambios que han observado en las prácticas de quienes han participado en la formación o se pueden recoger evidencias, huellas del trabajo, que muestren la efectiva aplicación de los aprendizajes adquiridos en un contexto formativo. Parece claro que la última opción, basada en productos, posee mayor objetividad que las meras opiniones pero también resulta difícil acotar hasta qué punto dicha producción es fruto únicamente de la formación desarrollada y por ello hay que huir de modelos causales excesivamente simplistas.

En cualquier caso, la transferencia depende, en parte, del diseño de la formación, aspecto en el que sí se puede intervenir directamente. Tal y como señala Iranzo (2009, p. 95), en 1988, Bolam estudió los diferentes niveles de impacto o cambios que se producían en la enseñanza en función del tipo de actividades que se realizan en un curso hallando que: explicar qué hacer posee un impacto bajo; hacer demostraciones posee un nivel medio y observar el trabajo real y ofrecer una retroacción al respecto tiene un alto nivel de impacto. Eso es precisamente lo que lleva a Marcelo (1994) a proponer el coaching como estrategia para facilitar el desarrollo de nuevas destrezas de enseñanza por parte de los 
profesores puesto que tiene una función principal: facilitar la transferencia de aprendizaje bajo los principios enunciados por Ellis (1965) de:

- Similitud de la tarea: La transferencia es mayor cuando las condiciones del entrenamiento son muy similares a las condiciones de la práctica. Ello supone que puede haber más transferencia cuando en las sesiones de formación se introducen elementos de práctica.

- Práctica y transferencia: Cuanto mayor cantidad de práctica exista sobre una tarea, mayor es la probabilidad de que se produzca un transfer positivo.

- Variedad de tareas o estímulos: En general, la variedad de las tareas, o de sus componentes incrementa la cantidad de transferencia. Ello supone la necesidad de mostrar a los profesores desde diferentes vías y con diferentes aplicaciones las destrezas en cuestión.

- Comprensión y transferencia: La transferencia es mayor si el sujeto comprende e interioriza las reglas o principios que son más apropiados a la resolución de un problema relacionado con las destrezas aprendidas.

En general, se obtienen mejores resultados en el aprendizaje de nuevas tareas después de haber sido entrenados previamente en una tarea similar. Las personas suelen confiar en su experiencia y conocimiento y adaptan estrategias que han resultado exitosas con anterioridad en las nuevas situaciones. Sin embargo, trasladar ciertos patrones a tareas que, por su complejidad, poseen características propias, puede no ser apropiado. El modelaje ha de adaptarse y una persona se hace competente cuando calibra y sopesa sus conocimientos, recursos y experiencias y, tras problematizar una situación compleja, moviliza sus saberes de forma pertinente y ajustada al contexto. Por ello valorar si se produce la transferencia requiere de contextos distintos y de circunstancias diferentes, para evitar aplicaciones mecánicas o simplistas.

\section{3. ¿Qué no es transferencia de la formación? Algunos ejemplos en la formación de formadores}

Como se acaba de señalar, no todas las evidencias que el participante en una acción formativa puede desarrollar pueden considerarse ejemplos de transferencia. Veamos algunos ejemplos que fueron debatidos en la I Jornada Internacional de Evaluación de la Transferencia de la formación celebrada en Barcelona el 26 de noviembre de 2014 (Red iberoamericana para el desarrollo de una plataforma tecnológica de soporte a la evaluación de los procesos de formación, RIDEFOR, Red 512RTo443 de la CYTED).

Ejemplo primero:

En una modalidad de formación en la que se realizan varias sesiones separadas en el tiempo y entre una y otra, el formado/a solicita una actividad práctica (el desarrollo de una unidad, un documento, una propuesta) ajustada a la realidad del aula o centro de cada uno.

No puede considerarse transferencia porque la actividad formativa está en curso aún y el hecho de realizar esa actividad puede, en ocasiones, entenderse como parte de las evidencias exigidas para acreditar el curso y, además, se suele someter al juicio experto del formador/a, que asesora y puede valorar/validar esas prácticas.

Como indica Herring (2012, pp. 4-5), las definiciones tradicionales tienden a centrar la transferencia en la repetición pero más recientemente se concibe como la capacidad de emplear ciertos aprendizajes en momentos posteriores en el tiempo y, por ello, no puede 
hablarse de transferencia si la actividad formativa está en curso y la aplicación se toma como una tarea obligatoria o recomendada por el formador entre sesión y sesión formativa.

Ejemplo segundo:

En una actividad formativa el objetivo es que los asistentes aprendan a elaborar un plan de comunicación interna, un plan estratégico, un plan de acogida,... y la formación se plantea de modo que acabe con un producto. Al curso siguiente, en sus respectivos centros aplican el plan elaborado. ¿्Puede ello considerarse prueba de transferencia?

No puede considerarse transferencia porque el objetivo de aprendizaje era aprender a elaborar cierto documento. Si esas personas son capaces de elaborar documentos en otros contextos, adaptándolos a las características de cada institución, ésa sí sería una muestra de transferencia pero el hecho de aplicar lo que elaboraron durante la formación no demuestra su capacidad para la elaboración autónoma en otros contextos.

Como indica el documento elaborado por los ICEs, hay que garantizar que la formación incorpore objetivos de transferencia, es decir, que se concrete a priori cuáles son los cambios que se esperan en el centro y en el profesorado una vez realizada la formación. (SGFiDPD, 2010, p. 12)

Ejemplo tercero:

Si un grupo de docentes realiza una formación sobre fomento del pensamiento crítico y al curso siguiente se mide la competencia de pensamiento crítico de los estudiantes, ¿̨es eso una medida de transferencia?

No puede considerarse transferencia porque en todo caso sería una medida de impacto, si pudiese atribuirse cierta mejora en el pensamiento crítico de los estudiantes a un cambio generado por la actividad formativa. Sin embargo, indirectamente podría ser también una medida de transferencia dada la dificultad existente para medir la aplicación de habilidades de pensamiento crítico, como ilustra el trabajo de ra-Ting (2012), quien ha explorado la transferencia de habilidades de pensamiento crítico a las prácticas del aula y sus efectos sobre el rendimiento de los estudiantes de séptimo y octavo. Algunos de estos estudiantes fueron asignados al azar como al grupo experimental (pensamiento crítico integrado) o al tradicional. Los resultados demostraron que los maestros lograron desarrollar con éxito el pensamiento crítico en ambos casos, es decir, que no es posible hallar diferencias significativas entre quienes siguieron un programa para adquirir y aplicar habilidades de pensamiento crítico y quienes no lo hicieron. Además de la reflexión sobre la dificultad de evaluar ciertos tipos de aprendizajes, la referencia al anterior trabajo constituye un ejemplo de que si se evalúan niveles superiores en la escala de Kirkpatrick, ya se puede inferir que se dieron niveles anteriores: por ejemplo, si se evalúa la transferencia es que se produjo aprendizaje -siempre que la transferencia no sea mecánica ni descontextualizada- y posiblemente no se hace necesario disponer de una evaluación de aprendizaje o, como en este caso, puede suceder que si se evalúa el impacto, ya se comprueba si hubo transferencia puesto que no puede haber cambios derivados de la aplicación si ésta no se ha producido.

Sirvan estos tres casos para ilustrar algunas de las dificultades más usuales en la conceptualización de la transferencia: confundirla con el aprendizaje o con el impacto o considerar ejemplos de transferencia ciertas prácticas sin disponer de evidencias que permitan imputarlas causalmente a la formación. Ante las dificultades narradas, algunos autores en los últimos años se han dedicado a analizar los factores que pueden favorecer u obstaculizar la transferencia de la formación, que es el objeto del estudio que se presenta a continuación.

\section{Método}

El estudio, realizado desde un enfoque cualitativo, muestra una investigación descriptiva de los factores que influyen en la transferencia de la formación. El estudio está basado en 
el análisis documental, con el que, por comparación, se identifican las categorías más reiterativas.

La selección de las contribuciones sobre transferencia de la formación que habían tratado los factores que pueden resultar impulsores de la misma se realizó en base a diferentes criterios según se tratase de estudios genéricos o específicos de formación del profesorado. Los estudios genéricos fueron escogidos combinando el número de citas que aparecían en Google Académico y el hecho de que perteneciesen a décadas diferentes, por si podía observarse algún cambio evolutivo. Así, se escogieron los estudios de Baldwin y Ford (1988), con 2798 citas: de Ford y Weissbein (1997), con 642 citas; de Holton (2005), con 227 citas; y de Blume Ford, Baldwin y Huang (2009), con 421 citas. Respecto a los dos estudios específicos relativos a la transferencia de la formación del profesorado, estos fueron elegidos a partir del número de citas pero realizando una búsqueda refinada que combinase transferencia y práctica profesional docente en educación superior. De modo que la búsqueda con los términos "Factors", "Professional Development" y "Teachers Practice" arrojó como principal resultado el estudio de Ingvarson, Meiers y Beavis (2005), con 438 citas y la búsqueda "Transfer of training", Workplace, Teacher Professional Development, y Higher Education arrojó como principal resultado, con 35 citas, el trabajo de De Rijdt, Stes, van der Vleuten y Dochy (2013).

Las aportaciones de estos trabajos fueron analizadas intentando hallar los elementos de coincidencia y categorizándolos en base a sus similitudes tomando como referente la propuesta de Baldwin y Ford (1988), que sugiere la triple división de los factores entre aquellos relativos a los participantes, al diseño y al ambiente de trabajo. Al observar que el análisis de los estudios arrojaba una cierta reiteración, se consideró no ser necesario el análisis de nuevos documentos que probablemente aportarían pocas novedades a datos ya saturados.

\section{Resultados}

\subsection{Sistematización de los factores influyentes en la transferencia de la formación}

El análisis pormenorizado de las contribuciones estudiadas se muestra en la tabla siguiente, en la que, se sitúan horizontalmente los factores que pudieran considerarse equivalentes en tanto que hacen referencia a la misma tipología de factores influyentes sobre la transferencia. Finalmente, éstos son agrupados en una categoría de orden superior cuya etiqueta (genérica, que emana del estudio de Baldwin y Ford, 1988, y de la coincidencia entre los nombres otorgados por los diversos autores y que se inicia en los tres casos como "Factores relativos a...") se ha colocado en la columna izquierda (tabla $1)$.

\subsection{Clasificación de los factores influyentes en la transferencia de la formación}

Como sintetizan Cordero y Ornelas (2014), los principales meta-análisis sobre transferencia de la formación vienen dadas por la revisión de estudios empíricos realizada por Baldwin y Ford (1988), por la comparación realizada por Blume et al. (2009) y por la investigación desarrollada por De Rijdt et al. (2013) sobre la transferencia en contextos de desarrollo profesional. 
Tabla 1. Clasificación de los factores relativos a la transferencia según los diversos modelos.

\begin{tabular}{|c|c|c|c|c|c|c|}
\hline & \multicolumn{4}{|c|}{ GENÉRICOS } & \multicolumn{2}{|c|}{ APLICADOS A FORMACIÓN DEL PROFESORADO } \\
\hline & $\begin{array}{l}\text { Baldwin y Ford } \\
\text { (1988) }\end{array}$ & $\begin{array}{l}\text { Ford y } \\
\text { Weissbein } \\
\text { (1997) }\end{array}$ & Holton (2005) & $\begin{array}{l}\text { Blume Ford, Baldwin } \\
\text { y Huang (2009) }\end{array}$ & $\begin{array}{l}\text { Ingvarson, Meiers y Beavis } \\
\qquad(2005)\end{array}$ & $\begin{array}{l}\text { De Rijdt, Stes, van der Vleuten } \\
\text { y Dochy (2013) }\end{array}$ \\
\hline 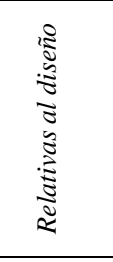 & $\begin{array}{l}\text { Factores previos a la } \\
\text { formación (el diseño de la } \\
\text { formación por una parte) }\end{array}$ & $\begin{array}{l}\begin{array}{l}\text { Diseño de la } \\
\text { formación }\end{array} \\
\text { Caract. de la } \\
\text { formación }\end{array}$ & $\begin{array}{l}\text { Elementos de habilidades: Diseño del proceso de } \\
\text { transferencia }\end{array}$ & & $\begin{array}{l}\text { Factores contextuales (el } \\
\text { soporte escolar), } \\
\text { Características estructurales } \\
\text { de los programas (la duración) } \\
\text { Características procesuales (el } \\
\text { seguimiento y feedback a los } \\
\text { estudiantes, las metodologías } \\
\text { activas) }\end{array}$ & $\begin{array}{l}\text { Diseño de la intervención: } \\
\text { análisis de necesidades, objetivos } \\
\text { de aprendizaje, estrategias de } \\
\text { autogestión, soporte tecnológico, } \\
\text { naturaleza o contenido del } \\
\text { programa, duración de la } \\
\text { formación, clima de } \\
\text { aprendizaje... } \\
\end{array}$ \\
\hline 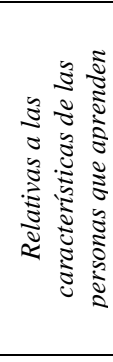 & $\begin{array}{l}\text { Factores previos a la } \\
\text { formación (las } \\
\text { características del } \\
\text { alumnado) Factores } \\
\text { posteriores a la formación } \\
\text { (el aprendizaje y la } \\
\text { retención) } \\
\text { Y factores que condicionan } \\
\text { la transferencia, como son } \\
\text { la generalización y el } \\
\text { mantenimiento }\end{array}$ & & $\begin{array}{l}\text { Elementos de habilidades: Capacidad personal } \\
\text { para transferir } \\
\text { Elementos Motivacionales: Motivación para } \\
\text { transferir. Esfuerzo/acción de transferencia } \\
\text { Resultados en la actuación } \\
\text { Elementos secundarios: Autoconfianza en la } \\
\text { habilidad de actuar en el local de trabajo } \\
\text { Preparación para aprender }\end{array}$ & $\begin{array}{l}\text { Autoeficacia previa a la } \\
\text { formación y posterior a } \\
\text { la formación } \\
\text { Motivación } \\
\text { Evidencias de } \\
\text { aprendizaje tras la } \\
\text { formación }\end{array}$ & & $\begin{array}{l}\text { Características de las personas } \\
\text { que aprenden: habilidad } \\
\text { cognitiva, autoeficacia, } \\
\text { motivación, personalidad, locus } \\
\text { de control, percepción de utilidad, } \\
\text { experiencia,... }\end{array}$ \\
\hline 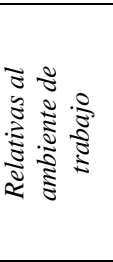 & $\begin{array}{l}\text { Factores previos a la } \\
\text { formación (entorno de } \\
\text { trabajo) }\end{array}$ & $\begin{array}{l}\text { Ambiente de } \\
\text { trabajo }\end{array}$ & $\begin{array}{l}\text { Elementos de habilidades: Oportunidad de } \\
\text { utilizarlos recursos necesarios para transferir. } \\
\text { Elementos Contextuales: Retroalimentación. } \\
\text { Suporte de los compañeros. Suporte de los } \\
\text { superiores. Abertura al cambio. Resultados } \\
\text { personales positivos. Resultados personales } \\
\text { negativos. Sanciones de los superiores. Validez de } \\
\text { los contenidos de formación en el local de trabajo }\end{array}$ & Ambiente de trabajo & & $\begin{array}{l}\text { Ambiente de trabajo: vinculación } \\
\text { estratégica, apoyo, clima de } \\
\text { transferencia, oportunidad de } \\
\text { aplicación, rendimiento de } \\
\text { cuentas. }\end{array}$ \\
\hline
\end{tabular}


Las variables que influyen en la transferencia detectadas por Baldwin y Ford (1988) en torno a tres grupos de factores (diseño de la formación, características del aprendiz y entorno de trabajo) se han ido enriqueciendo con los estudios posteriores que han sido analizados pero en líneas generales, se mantienen los factores relacionados con esos tres bloques considerados en la tabla anterior:

- Diseño de la formación (contenidos de la formación, secuencia formativa, análisis de necesidades, estrategias de instrucción, metas de aprendizaje, relevancia del contenido).

- Características del aprendiz (motivación, personalidad, capacidades, autoeficacia, utilidad percibida, expectativas carrera profesional y compromiso con la institución).

- Ambiente de trabajo (apoyo de los superiores, apoyo de los iguales, oportunidades de usar lo aprendido, clima de transferencia, rendición de cuentas, feedback,...).

En primer lugar, Baldwin y Ford (1988), cuando se refieren al diseño de la formación, aluden a los principios de aprendizaje, a la secuencia y a los contenidos de la formación. En un sentido amplio, la categoría "Diseño de la formación" puede considerarse un condicionante o un "input" para la transferencia. Dentro de la misma pueden englobarse, tanto factores contextuales (el grado de apoyo que posee en la institución), como elementos relativos al análisis de necesidades del que emerge la propuesta formativa (análisis de competencias vs. de tareas; análisis de labores individuales vs. tareas en equipo, etc.) y como los factores propiamente de la planificación y programación de la formación (su modalidad, características, duración, metodologías, sistemas de evaluación formativa y sumativa que prevé, selección de contenidos del programa, etc.).

En segundo lugar, los estudios analizados también coinciden en que las características de los participantes en la formación (capacidades, personalidad, motivación,...) son un aspecto condicionante de la transferencia de la formación. La dificultad de intervenir sobre ellos no debe de llevar a no tenerlos presentes. Especialmente Holton (2005) destaca la capacidad personal para transferir, en la que incluye tanto los elementos motivacionales como los que denomina secundarios, entre los que halla la autoconfianza como la preparación para aprender. Respecto a la motivación, Naquin y Holton (2002) habían mostrado ya los efectos de la motivación sobre el rendimiento en el trabajo a través del aprendizaje, que suele ser un proceso social, por lo que insisten en la necesidad de tener presente la influencia positiva de la sociabilidad en los procesos de transferencia. Y, como se aprecia en la tabla 1, Blume Ford, Baldwin y Huang (2009) insisten en la importancia de la misma. Respecto a otros elementos también relativos a los participantes, Ford y Weissbein (1997) se habían referido especialmente a la metacognición, entendida como capacidad de los estudiantes para monitorear y regular sus estrategias de aprendizaje con el fin de maximizar el aprendizaje y el rendimiento. Posteriormente, este aspecto es tomado especialmente en cuenta por los estudios relativos a formación del profesorado, tanto de Ingvarson, Meiers y Beavis (2005) como de De Rijdt, Stes, van der Vleuten y Dochy (2013).

Finalmente, no únicamente la formación y los participantes sino también el ambiente de trabajo puede facilitar u obstaculizar la generalización y el mantenimiento. El hecho de que, en el entorno de trabajo, se disponga de oportunidades de usar lo aprendido, del 
apoyo de los superiores, del apoyo de los iguales y de un clima de transferencia y exigencia que lleve a rendir cuentas, resulta determinante. Excepto en el estudio de Ingvarson, Meiers y Beavis (2005), en todos los documentos analizados el ambiente de trabajo aparece como una dimensión, de lo que se desprende la importancia de que las organizaciones consideren dicho ambiente para que se dé la transferencia.

En conjunto, las características del aprendiz y el ambiente de trabajo (bloques b y c de la tabla 1) influyen directamente sobre los factores posteriores a la formación, tanto sobre el aprendizaje como sobre la transferencia, mientras que el primer bloque, el relativo al diseño, influye directamente sólo sobre el aprendizaje y éste a su vez sobre la transferencia, con lo que su influencia sobre la transferencia es indirecta. El hecho de que dicha influencia no sea directa no significa que el diseño de la formación sea un elemento menos importante o secundario. Como ya hemos indicado anteriormente (Cano, 2015), la evaluación de los diseños formativos supondría probablemente una mejora de la eficiencia de las acciones formativas y un elemento esencial para el aseguramiento de la calidad.

\section{Conclusiones}

Las conclusiones de este estudio permiten comprender de forma más profunda la incidencia de diversos factores en la transferencia de la formación y, en consecuencia, resultan relevantes para orientar tanto futuros estudios sobre el tema como para generar ciertas orientaciones o lineamientos que permitan mejorar dicha transferencia. Estas orientaciones pueden ser útiles tanto para instituciones y unidades dedicadas a la formación, para que elaboren sus propuestas formativas considerando la ulterior transferencia, como para organismos y empresas en las que la formación se aplica, buscando el ambiente y apoyos que optimicen dicha aplicación.

Las conclusiones se estructuran en base a los tres ejes que han emergido como categorías básicas para la transferencia de la formación.

En primer lugar, respecto a los factores relativos al diseño, éstos incluyen todo el proceso y resultado del diseño. Esto significa que el hecho de que el diseño se realice concienzudamente puede influir positivamente en la transferencia. El programa de las Naciones Unidas para el Desarrollo vincula el diseño de programas directamente a la transferencia:

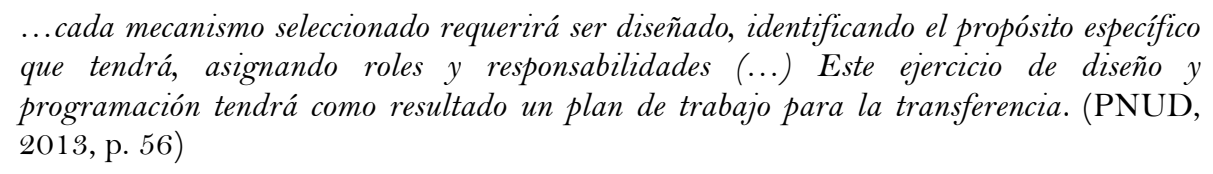

Para lograr que el diseño sea ajustado y transferible éste debe de basarse en evaluaciones diagnósticas de la situación y en un análisis de necesidades institucionales riguroso. Ello debe de suscitar una reflexión por parte de los responsables de las instituciones proveedoras de formación respecto a cómo se atiende a las necesidades formuladas por diversos colectivos de educadores y cómo éstas deben de poseer cierto grado de elaboración, una articulación con las políticas institucionales y una alineación entre todos sus componentes.

Por otra parte, las características del diseño (la duración, la naturaleza de los contenidos, los resultados de aprendizaje establecidos, las metodologías que se emplean, el clima que 
se establece) constituyen aspectos determinantes, como se indica en la vinculación entre evaluación y esas variables propuesta por Cordero, Cano, Benedito, Lleixà y Luna (2014). En síntesis, la naturaleza de las competencias que se desea promover, la finalidad de la modalidad formativa, las condiciones (financieras, temporales, de volumen de población participante en la formación, etc.) han de sopesarse para diseñar programas y acciones formativas que contribuyan a la mejora, aunque quizás todas ellas no permiten la transferencia, al menos inmediata.

En segundo lugar, respecto a los factores relativos a las características de las personas que aprenden, los autores analizados insisten en la importancia de la capacidad de aprendizaje, de la motivación, de la autoeficacia, tanto antes como después de la formación y de la percepción de utilidad. Ello sugiere que, pese a tratarse de aspectos personales en los que es más difícil incidir, establecer políticas que contribuyan a mejorar estas variables indirectas podría redundar significativamente en mejorar la transferencia. Esta aportación ha sido reiterada por Holton (2005) y la investigación muestra que tanto en entornos laborales (Awais y Kaur, 2010) como en entornos académicos (Prieto, 2008; Zabalza, 2011), la autoeficacia resulta un factor altamente determinante de la transferencia y subrayan, por tanto, la conveniencia de estimularla.

Finalmente entre los factores relativos al ambiente de trabajo destacan aquellos que aconsejan disponer los mecanismos que aseguren el apoyo de los superiores y que faciliten el soporte de los compañeros, así como facilitar la oportunidad para aplicar los conocimientos. En este sentido, las modalidades de formación en centros acompañadas de un liderazgo decidido que facilite ese apoyo tanto por parte de la dirección como entre iguales son, probablemente, las que llevan a una mayor posibilidad de transferencia (Sanmartí y Masip, 2011). Esta idea es reforzada por Murillo y Krichesky (2012) al indicar que es necesario implementar cambios estructurales que promuevan y acompañen la mejora de la práctica de enseñanza y contar con un elenco adecuado de profesionales que faciliten el cambio y/o profesores que funcionen a modo de asesores. El apoyo de superiores e iguales y la instauración de medidas estructurales que posibiliten la transferencia son elementos indispensables para transferir. Este apoyo tiene características particulares en los entornos académicos, diferenciales respecto a los entornos empresariales, por lo que este factor debe ser abordado con cautela.

A partir de los análisis indicados parece que existen algunas lecciones aprendidas que pueden ayudar a que la transferencia de la formación sea posible y la evaluación de la misma constituya un proceso viable. Se detallan a continuación a modo de decálogo:

1. Diseñar la formación de modo que finalice con un proyecto, de modo que los aprendizajes deban ser plasmados en un producto

Quizás no sea siempre posible puesto que la naturaleza de los diversos contenidos de la formación puede hacer inviable que se apliquen en un documento final. Sin embargo, como ya indicó Marcelo (1994) la integración teoría y práctica en forma de propuestas elaboradas por cada participante o equipo de participantes puede contribuir a que se traslade ese aprendizaje a futuros contextos y coyunturas.

\section{Diseñar la evaluación desde el momento inicial, cuando se planifica la formación}

Entre diseño y evaluación hay un efecto trade-off, es decir, cuanto más tiempo y dinero se invierte en diseñar, menos dificultades presenta la realización de la evaluación de una actividad formativa (García Montalvo, 2008). 
3. Extender marcos temporales y dotar la formación de un sistema de seguimiento, atendiendo a los plazos para evaluar la transferencia

Como indican Boqué y García (2010) o la propia ACNUDH (2010) para referirse a la evaluación del impacto que dicha transferencia genera sobre la organización, el lapso temporal debe de extenderse, en cuyo caso los resultados últimos del programa de formación requieren de un seguimiento longitudinal y superan los marcos temporales con los que las personas que financian los programas suelen trabajar. Los resultados de Ingvarson, Meiers y Beavis (2005) refuerzan esto con su estudio en el que hallaron, mediante análisis de regresión, la significatividad no sólo de la duración de un programa sino de su extensión en el tiempo, así como la relevancia de disponer de un sistema de seguimiento.

\section{Identificar y escribir separadamente los objetivos de aprendizaje y los objetivos de transferencia}

Es importante diferenciar qué se espera que aprenda cada persona o colectivo y qué se espera que cambien en sus respectivos puestos de trabajo (Iranzo, 2012). La lógica que lleva a pensar en la formación con la esperanza de que conduzca a cambios debe de ser revertida (Paricio, 2013) y partir de los resultados que se desean obtener en la institución para, a partir de los mismos, determinar qué debe de realizarse y, en consecuencia, qué formación es necesaria.

\section{Revisar la instrumentación disponible}

La evaluación de la transferencia únicamente puede realizarse, de modo indirecto, a partir de opiniones o, de modo directo, a partir de evidencias. Disponer de un conjunto de instrumentos que puedan ayudarnos a recopilar esas informaciones (como los disponibles en la plataforma www.ridefor.net) y de experiencias (Cano y Bartolomé, 2014) que muestren cómo los han utilizado puede resultar de gran ayuda.

\section{Habilitar contenedores de evidencias de transferencia}

Siempre que sea posible porque el diseño formativo lo ha previsto y porque se dan los recursos materiales y temporales imprescindibles, es deseable basar la evaluación en datos más que en opiniones. Puede ser interesante explorar las posibilidades de la tecnología para recoger y visibilizar las evidencias de transferencia desarrollando blogs o fotologs y/o incorporando huellas del trabajo en portafolios electrónicos (Barberá, Baustista, Espasa y Guasch, 2006). Además de Mahara o Eduportfolio, se puede disponer de contenedores compartidos en Google Sites o Dropbox o en cualquier LAMS y ello puede hacer más viable el almacenamiento, gestión y evaluación de las evidencias de transferencia, documentando el desarrollo o progresión en un proceso de aplicación de la formación y reflexionando sobre el mismo.

\section{Comprometer a los agentes participantes}

En el caso de los centros de educación no universitaria correspondería a los propios participantes, al formador y a la inspección (Antúnez, 2009), pero es el equipo directivo quien debiera comprometerse en dinamizar la transferencia y en evaluarla junto a los protagonistas. Sin embargo, esta necesidad de seguimiento por parte de diversos agentes topa con la cultura individualista denunciada por Manzanares y Galván (2012), quienes detectan que cualquier agente externo al centro se percibe como un intruso lo cual dificulta la evaluación de la transferencia de aprendizajes al centro o al aula. 


\section{Identificar y promover los factores que facilitan la transferencia}

Como se ha recogido de Baldwin y Ford (1988), la generalización hace referencia a cómo los aprendizajes o las competencias alcanzadas tras el proceso formativo pueden manifestarse en el puesto de trabajo y pueden ser aplicados a situaciones y coyunturas distintas a las del programa de formación. Por su parte, el mantenimiento, en cambio, se refiere a la amplitud en el tiempo en el que las competencias adquiridas continúan siendo empleadas con efectividad en el puesto de trabajo. La institución y los diseñadores de la formación deben de plantearse qué estrategias son necesarias para que se den ambos factores. Estas estrategias, como se ha indicado, pueden incidir en dimensiones más personales (motivación, autoeficacia, locus de control,...) o en dimensiones más institucionales.

\section{Identificar y minimizar las barreras a la transferencia}

El estudio de Doherty (2011) analiza la transferencia de la formación (de dos tipos de seminarios de trabajo de formatos diferentes acerca de las herramientas de la web 2.0) sobre la docencia con docentes universitarios en la Universidad de Auckland. Pese a que muestran altas tasas de satisfacción y de aprendizaje, muy pocos aplicaron aquello que aprendieron. En las entrevistas realizadas tres meses después de haber tenido lugar la formación, aceptan esa falta de transferencia, pero no son capaces de explicar claramente los motivos para la misma. Doherty (2011) considera que las barreras a la transferencia pueden ser de tipo psicológico (falta de motivación y de reconocimiento o recompensa), organizativo (falta de provisión de apoyo y, especialmente, de una temporalización adecuada), político (carencia de una estrategia institucional) y cultural (existencia de valores y normas que no facilitan el cambio).

\section{Evaluar el impacto}

Es posible que no todos los niveles deban de ser evaluados. Del mismo modo en que el aprendizaje, en ciertas modalidades formativas, no es necesario que sea evaluado puesto que si disponemos de evidencias de transferencia podemos inferir que se produjo aprendizaje, es posible también que si disponemos de indicadores de impacto podamos dar por supuesta la transferencia. En cualquier caso, el objetivo de transferir es, en última instancia, lograr el impacto en el conjunto de la organización y/o en los usuarios últimos, por lo que, en caso de no poder evaluar todo, se pueden centrar los esfuerzos en evaluar lo más relevante.

Hay que hacer notar que de las diez propuestas que se acaban de señalar, la octava y la novena tienen un carácter genérico y la séptima hace referencia básicamente al tercer tipo de factores, relativos al ambiente de trabajo. El resto, sin embargo, son todas ellas propuesta referidas al diseño, tanto de la formación (primera, cuarta) como de la evaluación (segunda, tercera, quinta, sexta y décima). Desde las instituciones dedicadas a la capacitación ése es, sin duda, el campo de intervención plausible. De ahí deriva la necesidad de insistir en la calidad de los diseños formativos.

La evaluación de la transferencia resulta, por todo lo indicado, un proceso complejo. Requiere del compromiso de las instituciones promotoras de la formación para dotar de recursos temporales y personales que permitan realizar dicha evaluación; requiere de formadores implicados desde el diseño hasta el seguimiento post-actividad formativa; requiere de una cultura de corresponsabilidad de los participantes y de un alineamiento de los programas formativos con los objetivos institucionales, aspectos todos ellos, 
difíciles de lograr. Finalmente se requieren avances en la investigación sobre transferencia de la formación para complementar y ajustar los factores que la literatura ha hallado relativos al diseño, a las características de los participantes y al ambiente de trabajo a la formación del profesorado, lo cual supone un reto sobre el que trabajar en futuras investigaciones. Dichas investigaciones pueden ahondar en estudios descriptivos como el que se ha realizado y cuyas limitaciones vienen derivadas tanto de la propia naturaleza de un estudio descriptivo basado análisis documental como de la selección de los estudios que se ha realizado. Futuras investigaciones podrían ampliar los estudios analizados, incorporando nuevos descriptores en la búsqueda e incorporando los documentos más recientes. Por otra parte, en el futuro sería interesante disponer de investigaciones de desarrollo longitudinal, que serían especialmente relevantes para este objeto de estudio.

\section{Referencias}

ACNUDH (2010). Cómo evaluar las actividades de capacitación en derechos humanos. Ginebra: Oficina del Alto Comisionado de las Naciones Unidas para los Derechos Humanos.

Antúnez, S. (2009). La inspección educativa y la evaluación de la formación permanente de los profesionales de la educación. Avances en Supervisión Educativa, 10, art 6.

Awais Bhatti, M. y Kaur, S. (2010). The role of individual and training design factors on training transfer. Journal of European Industrial Training, 34(7), 656-672. doi: 10.1108/03090591011070770

Baldwin, T.T. y Ford, J.K. (1988). Transfer of training: A review and directions for future research. Personnel Psychology, 41(1), 63-105. doi: 10.1111/j.1744-6570.1988.tbo0632.x

Barberà, E., Bautista, G., Espasa, A. y Guasch, T. (2006). Portfolio electrónico: desarrollo de competencias profesionales en la Red. RUSC. Revista de Universidad y Sociedad del Conocimiento, 3(2), 55-66.

Blume, B., Ford, K., Baldwin, T. y Huang, J. (2009). Transfer of training: a meta-analytic review. Journal of Management, 36(4), 1065-1 105. doi: 10.1177/0149206309352880

Bonal, X. (2015). Crisis, educación y desigualdad: una cuestión de paradojas. Investigar em Educação, 2(3), 23-32.

Boqué, M.C. y García, L. (2010). Evaluación diferida de la formación del profesorado en convivencia y mediación. Revista Electrónica Interuniversitaria de Formación del Profesorado, $13(3), 87-94$.

Cano, E. (2015). Evaluación de la formación: algunas lecciones aprendidas y algunos retos de futuro. Educar, 51(1), 109-125. doi: 10.5565/rev/educar.684

Cano, E. y Bartolomé, A. (Coords.) (2014). Evaluar la formación es posible. Evaluación de la transferencia de la formación de formadores con soporte tecnológico. Barcelona: Transmedia XXI (LMI-UB).

Cordero, G. y Ornelas, D. (noviembre, 2014). Aportes de la investigación educativa a la evaluación de la transferencia de la formación. Comunicación presentada en I Jornada Internacional de Evaluación de la Transferencia de la Formación. Barcelona.

Cordero, G., Cano, E., Benedito, V., Lleixà, T. y Luna, E. (2014). La planificación y la evaluación de la formación pedagógica del profesorado universitario: orientaciones para su definición institucional. Barcelona: ICE. 
De Rijdt, C., Stes, A., van der Vleuten, C. y Dochy, F. (2013). Influencing variables and moderators of transfer of learning to the workplace within the area of staff development in higher education: research review. Educational Research Review, 8, 48-74. doi: 10.1016/j.edurev.2012.05.007

Doherty, I. (2011). Evaluating the impact of professional development on teaching practice: Research findings and future research directions. US-China Education Review, 5, 703-714.

Ellis, H. (1965). The transfer of learning. Nueva York: The MacMillan Company.

Eurydice (2006). La evaluación de la calidad de la formación del profesorado europeo. Bruselas: Eurydice.

Evans, L., Hodkinson, P. y Unwin, L. (Eds.). (2002). Working to learn: transforming learning in the workplace. Londres: Routledge.

Evans, L., Hodkinson, P., Rainbird, H. y Unwin, L (2006). Improving workplace learning. Londres: Routledge.

Feixas, M. (Coord.). (2013). Informe de investigación: Factores de transferencia de la formación docente Recuperado de: http://www.red-u.org/

Feixas, M. (Coord.). (2015). Monográfico sobre impacto y transferencia de la formación docente universitaria. Educar, 51(1), 45-67.

Feixas, M., Fernández, A., Lagos, P., Quesada, C. y Sabaté, S. (2013). Factores condicionantes de la transferencia de la formación docente en la universidad: un estudio sobre la transferencia de las competencias docentes. Infancia y Aprendizaje, 36(3), 401-416. doi: 10.1174/021037013807533034

Fernández, I., Guisasola, G., Garmendia, M., Alkorta, I. y Madinabeitia, A. (2013). ¿Puede la formación tener efectos globales en la universidad? Desarrollo docente, metodologías activas y currículum híbrido. Infancia y Aprendizaje, 36(3), 387-400. doi: $10.1174 / 021037013807532990$

Ford, J. y Weissbein, D. (1997). Transfer of training: an updated review and analysis. Performance Improvement Quarterly, 1O(2), 22-41. doi: 10.1111/j.1937-8327.1997.tb00047.x

Gairín, J. (2010). La evaluación del impacto en programas de formación. REICE. Revista Iberoamericana sobre Calidad, Eficacia y Cambio en Educación, 8(5), 19-43.

García Montalvo, J. (2008). La ayuda al desarrollo: su eficacia y métodos experimentales para su evaluación En J. García Montalvo (Ed.), El análisis experimental de la ayuda al desarrollo: lo que funciona y lo que no funciona (pp. 17-48). Bilbao: Fundación BBVA.

Haskell, R. (2001). Transfer of learning: Cognition, instruction and reasoning. San Diego, CA: Academic Press.

Haverila, M., Myllylä, M. y Torp, H. (2009). Towards innovative virtual learning in vocational teacher education: narratives as a form of meaningful learning. Recuperado de: http://www.eurodl.org/materials/contrib/2009/Haverila_Myllyla_Torp.pdf

Herring, J. (2012). Year 7 students, information literacy, and transfer: a grounded theory. Chicago, IN: American Library Association.

Holton, E.F. (1996). The flawed four-level evaluation model. Human Resource Development Quarterly, 7(1), 5-23.

Holton, E.F. (2005). Holton's evaluation model: new evidence and construct elaborations. Advances in Developing Human Resources, 7(37), 37-54. doi: 10.1177/1523422304272080 
Iranzo, P. (2009). Innovando en educación. Formarse para cambiar: un viaje personal. Barcelona: Erasmus Ediciones.

Iranzo, P. (2012). Asesoramiento pedagógico al profesorado. Madrid: Síntesis.

Ingvarson, L., Meiers, M. y Beavis, A. (2005). Factors affecting the impact of professional development programs on teachers' knowledge, practice, student outcomes \& efficacy. Education Policy Analysis Archives, 13(10), 1-26. doi: 10.14507/epaa.v13n 10.2005

Kirkpatrick, D. y Kirkpatrick. J. (2000). Evaluación de acciones formativas: los cuatro niveles. Barcelona: Epise.

Kirkpatrick, J. y Kirkpatrick, W. (2010). ROE's rising star: why return on expectations is getting so much attention. Recuperado de: http://www.astd.org/Publications/Magazines/TD/

Manzanares, A. y Galván, M.J. (2012). La formación permanente del profesorado de educación infantil y primaria a través de los centros de profesores. Un modelo de evaluación. Revista de Educación, 359, 431-455.

Marcelo, C. (1994). Formación del profesorado para el cambio educativo. Barcelona: EUB.

Melgarejo, J. (2013). Gracias, Finlandia: lo que podemos aprender del sistema educativo de más éxito. Barcelona: Plataforma.

Murillo, F.J. (2003). El movimiento teórico-práctico de mejora de la escuela. Algunas lecciones aprendidas para transformar los centros docentes. REICE. Revista Iberoamericana sobre Calidad, Eficacia y Cambio en Educación, 1(2), 7-23.

Murillo, F.J. y Krichesky, G.J. (2012). El proceso del cambio escolar. Una guía para impulsar y sostener la mejora de las escuelas. REICE. Revista Iberoamericana sobre Calidad, Eficacia y Cambio en Educación, 1O(1), 26-43.

Naquin, S.S. y Holton, E.F. (2002). The effects of personality, affectivity, and work commitment on motivation to improve work through learning. Human Resource Development Quarterly, 13, 357-376. doi: 10.1002/hrdq.1038

OCDE (2005). Teachers matter: attracting, developing and retaining effective teachers. París: OCDE.

Paricio, J. (2013). ¿Repensar la formación del profesorado? ¿Por qué habríamos de hacer tal cosa? Revista de la Red-U, 11(3), 56-78.

Parsons, D., Hill, I., Holland, J. y Willis, D. (2012). Impact of teaching development programmes in higher education. Londres: HEA Research Series.

Pineda, P. (2000). Evaluación de impacto de la formación en las organizaciones. Educar, 27, 119133.

Pineda, P. (2002). Evaluación de la formación en las organizaciones. En P. Pineda (Coord.), Gestión de la formación en las organizaciones (pp. 247-277). Barcelona: Ariel.

Pineda, P. (Coord.). (2011). Avaluació de la transferència de la formació a l'administració pública de Catalunya. Barcelona: Escola d'Administració Pública de Catalunya.

Pineda, P. (Dir.). (2012). Evaluación de la eficacia de la formación en la administración pública Española. Barcelona: Grupo EFI.

Pineda, P. y Sarramona, J. (2006). El nuevo modelo de formación continua en España: balance de unos años de cambio. Revista de Educación, 341, 705-736.

Pineda, P., Moreno, A., Úcar, X. y Belvis, E. (2008). Derecho a la calidad: evaluación de la formación permanente en el sector de la educación infantil en España. Revista de Educación, 347, 101-126. 
Pineda, P., Moreno, A., Úcar, X. y Belvis, E. (2010). Is continuing training useful for pre-school teachers? Effects of training on pre-school teachers and centers. European Early Childhood Educational Research Journal, 18(3), 407-421. doi: 10.1080/1350293x.2010.500081

Pineda, P., Belvis, E., Moreno, V., Duran, M. y Úcar, X. (2011). Evaluation of training effectiveness in the Spanish health sector. Journal of Workplace Learning, 23(5), 315-330. doi: $10.1108 / 13665621111141911$

PNUD (2013). Sistematización para transferir conocimiento. Recuperado de: http://webapp3docs.undp.org/procurement_notices/notice_doc_23192_765125625.pdf

Prieto, L. (2008). La enseñanza universitaria centrada en el aprendizaje. Barcelona: Narcea.

Quesada, C., Espona, B., Ciraso, A. y Pineda, P. (2015). La eficacia de la formación de los trabajadores de la administración pública española: comparando la formación presencial con el eLearning. Reforma y Democracia, 61, 107-132.

Sanfabián, J.L., Belver, J.L. y Álvarez, C. (2014). ¿Nuevas estrategias y enfoques de aprendizaje en el contexto del espacio europeo de educación superior? Revista de Docencia Universitaria Red-U, 12(4), 249-280.

Sanmartí, N. y Masip, M. (2011). ¿Cómo hacer que la formación impulse cambios en un centro? Aula de Innovación Educativa, 201, 10-14.

SGFiDPD (Coord.). (2010). Transferència de la formació en centre. Barcelona: ICE-Subdirecció General de Formació i Desenvolupament del Personal Docent.

Tejada, J. y Ferrández, E. (2007). La evaluación del impacto de la formación como estrategia de mejora en las organizaciones. Revista Electrónica de Investigación Educativa, 9(2), 167-189.

Tejada, J., Ferrández, E., Jurado, P., Mas, O., Navío, A. y Ruiz, C. (2008). Implicaciones de la evaluación de impacto: Una experiencia en un programa de formación de formadores. Bordón, 60(1), 163-183.

Ya-Ting, C.Y. (2012). Cultivating critical thinkers: Exploring transfer of learning from preservice teacher training to classroom practice. Teaching and Teacher Education, 28, 11161130. doi: 10.1016/j.tate.2012.06.007

Zabalza, M.A. (2011). Formación del profesorado universitario: mejorar a los docentes para mejorar la docencia. Educação, 36(3), 397-424. doi: 10.5902/198464442969 